\title{
Research and Development of an Augmented \\ Reality Visual Image-Guided Radiotherapy Positioning Technology
}

\section{Li Chunying}

Nanjing Medical University

\section{Lu Zhenda}

Nanjing Medical University

$\mathrm{He} \mathrm{Mu}$

Nanjing Medical University

Sui Jianfeng

Changzhou Second People's Hospital

\section{Lin Tao}

Changzhou Second People's Hospital

Xie Kai

Changzhou Second People's Hospital

\section{Sun Jiawei}

Changzhou Second People's Hospital

Ni Xinye ( $D$ nxy2000@aliyun.com )

Nanjing Medical University

\section{Research}

Keywords: augmented reality, radiotherapy, positioning of patients, three-dimensional visualization, guided radiotherapy

Posted Date: December 22nd, 2020

DOl: https://doi.org/10.21203/rs.3.rs-131843/v1

License: (c) (i) This work is licensed under a Creative Commons Attribution 4.0 International License. Read Full License 


\title{
Research and Development of an Augmented Reality Visual
}

\section{Image-Guided Radiotherapy Positioning Technology}

\author{
Li Chunying a,b,c, Lu Zhenda a,b,c, He Mu a,b,c, Sui Jianfeng a,b,c, Lin Tao a,b,c, Xie Kai a,b,c, Sun
}

Jiawei a,b,c, Ni Xinye a,b,c *

${ }^{a}$ Department of radiotherapy, Changzhou Second People's Hospital, Nanjing Medical University, China; ${ }^{b}$ Laboratory of medical physics center, Nanjing Medical University, China;

${ }^{c}$ Changzhou Key Laboratory of medical physics, China

Corresponding author at: Department of radiotherapy, Changzhou Second People's Hospital, China.

E-mail address: nxy2000@aliyun.com

\section{Abstract:}

Objective: This study is conducted to explore the development of a noninvasive and nonradiative guided radiotherapy positioning system based on augmented reality (AR) technology to increase positioning accuracy and completely eradicate positioning errors.

Method: The development system includes three monitors and supporting equipment. The 3D virtual model is obtained by 3D reconstruction of the radiotherapy plan data set and is superimposed on the correct treatment position of the real treatment environment by using augmented reality production software. This approach provides the standard for the patient's positioning and guides the patient. The position of the patient then coincides with the 3D virtual model through the visual image method, and positioning error of the radiotherapy position is reduced.

Results: A total of nine cases are included in this study, namely, three patients who require head and neck radiotherapy and six patients who require chest and abdomen radiotherapy. According to experimental results, no statistical difference exists between AR-guided positioning and manual positioning for patients requiring head and neck radiotherapy. However, AR-guided positioning can keep errors of $\mathrm{x}$-axis, $\mathrm{y}$-axis and z-axis within small ranges, such as -0.092-0.072, -0.052-0.228, and -0.192-0.05 cm, respectively. For patients requiring chest and abdomen radiotherapy, AR-guided positioning is not statistically different from manual positioning on the $\mathrm{x}$-axis, but statistical differences were found on the $\mathrm{y}$-axis and $\mathrm{z}$-axis. The errors of AR-guided positioning on $\mathrm{y}$-axis and z-axis are much smaller than those of manual positioning.

Conclusions: The AR visual image guided radiotherapy set-up system can increase radiotherapy positioning accuracy well, especially for patients requiring chest and abdomen radiotherapy.

Keywords: augmented reality, radiotherapy, positioning of patients, three-dimensional visualization, guided radiotherapy

\section{Introduction}

With the development of computer technologies, three-dimensional (3D) visualization technology plays an increasingly important role in people's daily life. As a 3D visualization technology, augmented reality (AR) technology is developing gradually.

AR technology is a branch of virtual reality (VR) technology. Different from VR, in which users are immersed completely in the virtual environment, AR integrates the virtual and real worlds. It generates a virtual environment with vivid visual, auditory force, and touching and motion sensations ${ }^{[1]}$. Users are immersed in the virtual environment through a sensing equipment, allowing direct natural interaction between users and the environment ${ }^{[2]}$. Mobile AR integrates intelligent display technology, registration tracking technology, virtual fusion technology[3], and man-machine interaction technology, thereby realizing seamless connection between 3D virtual object and real scenes. Mobile AR increases the perception range of people significantly. Currently, AR is widely used in various fields, such as education, navigation, games, and medicines. It possesses very promising application prospects in the medical field.

Although AR has been increasingly applied to the medical field, it is hardly used in radiotherapy around the whole world. In 2009, 
Talbot et $\mathrm{al}^{[4]}$ proposed the application of AR to external radiotherapy for the first time. However, this system is only used as a feasibility study. The 3D information only includes the external contour of the body surface. The camera cannot be blocked. The placement position has strict requirements.

At present, in addition to the traditional cone-beam CT (CBCT) and Electronic Portal Imaging Device (EPID)-assisted positioning methods, several new navigation methods have been applied to radiotherapy. Calypso electromagnetic navigation technology is used in the radiotherapy of prostate cancer, liver cancer, lung cancer and other diseases. It detects the electromagnetic signal fed back by beacon balise ${ }^{[5]}$ through electromagnetic array and measures the therapeutic target position in 3D space for the continuous tracking of the target area. However, it is an invasive operation[6], and the balise is prone to offset or no response[7]. Surface guided radiation therapy (sgrt) has been used in the treatment of breast cancer in recent years. It obtains the patient surface by optical projection ${ }^{[8][9]}$ and compares it with the reference surface that is generated or captured from planned CT data[10]. Combined with deep breath holding (DIBH) technology, sgrt can significantly reduce the radiation dose of breast cancer treatment center and can effectively protect the heart. However, some main problems exist. When the Gantry rotates to block a camera, the patient's surface data are lost. Positioning accuracy is also affected when the patient's skin is black or damaged.

This study aimed to explore the feasibility of using AR to improve the positioning accuracy of radiotherapy. Moreover, a noninvasive and nonradiative guided radiotherapy positioning system was developed. This system applied the 3D visualization technology to solve positioning errors caused by the invisibility of organs and tumor tissues, to decrease positioning error, and to completely eradicate the occurrence of treatment mistakes.

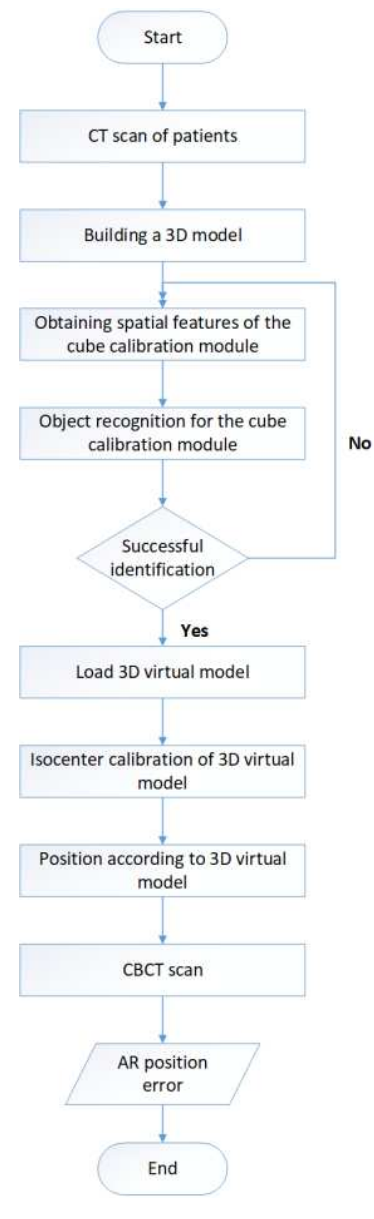

\section{Materials and methods}

\subsection{AR system}

The main process used in this study is shown in Fig. 1. The main process of this system is shown in Fig.1. It implemented 3D modeling to outer appearance and normal tissues, PTV, and CTV based on patient CT data. Meanwhile, a virtual cube with the same size as the cube calibration module was established in TPS and was located in the center of the LINAC. This virtual cube was modeled together with the patient data as a whole. The cube calibration module was aligned with the laser positioning line. The cube calibration module was identified by the AR object recognition technology, and the previously constructed 3D virtual models were loaded. By adjustment, the virtual cube in the 3D virtual model was in accordance with the cube calibration module in all directions. Later, the cube calibration module was removed, and patient positioning was performed. Monitors have in-built cameras for the real-time acquisition of images of patients on the treatment couch. When the patient was spatially anastomosed with the abovementioned 3D virtual model, especially the body surface contour. The treatment can be carried out only when the actual patient's body surface was highly compatible with the 3D virtual model .

\subsection{Patients}

In this study, nine patients who require radiotherapy were chosen as our research objects, including three patients requiring radiotherapy on the head and neck and six patients requiring radiotherapy on the chest and abdomen. Positioning errors of each patient in three conventional manual positioning performances and

three AR-guided positioning performances were collected. Informed consent was obtained from the subjects Fig1.Manufacturing process of the system in the human experiments, and the privacy rights of the subjects were respected.

\subsection{Acquisition of CT data and 3D modeling}

DICOM images were collected through the CT scanning of nine patients. CT data were input into the therapy planning system (TPS) to depict the profiles of target volume of radiotherapy and important protection of organs and tissues. Finally, the DICOM-RT dataset 
output was obtained and then input into the medical image processing software (3D Slicer version 4.11, National Institutes of Health); Open-source toolkit was applied for radiation therapy research to construct the 3D medical models. These constructed 3D medical models were input into 3D MAX (2018 version, Autodesk Company, USA) to adjust the model format. The virtual field was established according to dicom-RT field information.

\subsection{System setting}

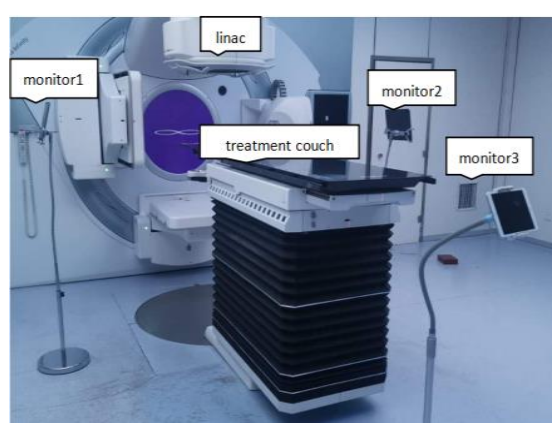

The hardware system included three monitors and supporting devices. The supporting devices were placed at the right, left, and end of the treatment couch (Fig. 2). The supporting devices were adjusted to the same height of the treatment couch, and three monitors were put in. The three monitors were connected online by using the multipeer-connectivity short-range communication technology to realize the real-time sharing of environmental information data. Three devices were fixed in the position shown in Fig. 2, and the virtual model was operated

Fig. 2 Overall system setting interactively in one device. The position of the virtual model in the other two devices was updated in real time with the interactive operation, thereby reducing the error due to device movement. If the rack rotates and blocks a certain device, the other two devices would also send information on the location of the virtual model. There would be no loss of model tracking.

\subsection{AR simulation}

The processed 3D visual model was input into the AR manufacturing software, and an AR interactive system was constructed. The main process was able to reproduce the real scenes through 3D modeling, and the object was driven in real-time to move in the 3D environment, thereby realizing AR simulation on mobile terminals. The main technologies used include visual-inertial odometry (VIO), inertial measurement unit (IMU) and simultaneous localization and mapping (SLAM). The system used AR object recognition technology to guide radiotherapy positioning. First, the reference object was scanned and the spatial feature information of the reference object was recorded in advance. When the system was used for 3D object recognition again, the image information obtained by the camera was compared with the space feature information of the reference object. After a successful comparison, the established 3D virtual model was loaded. The main steps of object recognition included feature point extraction, feature point matching, camera pose estimation and feature point location estimation.

\subsubsection{Feature point extraction and matching}

Object recognition feature points refer to the points with large difference in brightness, color, and grayness in video images obtain ed through vio. To ensure better real-time performance, ORB feature points were used for visual navigation in this system. The main process involved fast corner extraction and brief descriptor creation. As shown in Fig. 3 (a), fast corner extraction was performed to select pixel p from the image. Its brightness is $I_{P}$, and the threshold $T_{P}$ was set. Pixel $P$ was the center, and all pixels on the circle with radius of 3 are selected. If the brightness of $N$ consecutive points on the circle was greater than $I_{P}+T_{P}$ or less than $I_{P}-T_{P}$, then these points were considered as feature points. To reduce the influence of scale change and rotation change on feature point detection, the Image Pyramid and Intensity Centroid method were respectively constructed to ensure scale and rotation invariance. After extracting the key points, each key point was described by brief feature and expressed in binary form. After extracting the feature points, they were matched. The burden of the subsequent operations, such as pose estimation and optimization, were reduced only after the features in two adjacent frames were correctly matched. In Euclidean space, the two descriptors were expressed as follows: brief $_{1}[i]=\left\{x_{1}, x_{2}, \ldots x_{n}\right\}$, brief ${ }_{2}[i]=\left\{y_{1}, y_{2}, \cdots y_{n}\right\}$. The difference of descriptors in Euclidean space is $\operatorname{COV}\left(\right.$ brief $_{1}[i]$, brief $\left._{2}[i]\right)=\sum_{i=1}^{n} \sqrt{x_{i}^{2}-y_{i}^{2}}$. A smaller difference between the descriptors leads to a higher similarity between the two features, thereby achieving the matching of feature points. 


\subsubsection{Estimate camera pose}

After accurately matching the feature points of two adjacent frames, the motion of the camera was estimated according to the feature point pair, and the translation matrix $\mathrm{T}$ and rotation matrix $\mathrm{R}$ of the motion from the first frame to the second frame were solved. As shown in Fig. $3(b)$, the first frame image was $I_{1}$, the second frame image was $I_{2} . O_{1}$ and $\mathrm{O}_{2}$ were the center of the camera, and $\mathrm{p}_{1}$ and $\mathrm{p}_{2}$ were the feature points. These two points can be obtained by feature matching; they connecting $\overrightarrow{O_{1} P_{1}}$ and $\overrightarrow{O_{2} P_{2}}$ in3D space; and they intersect at point $\mathrm{p}$. The fundamental matrix $\mathrm{F}$ can be solved by simplifying the epipolar constraint formula: $p_{2}^{T} F p_{1}=0$, whereas ${ }^{F=} K^{-T} \mathrm{t}^{\wedge} R K^{-1}, \mathrm{~K}$ is the known camera internal parameter matrix, from which the camera rotation matrix $\mathrm{R}$ and translation matrix $\mathrm{t}$ can be decomposed to determine camera positioning. The world coordinate system of the system was obtained.

\subsubsection{Estimating the spatial position of feature points}

After acquiring the camera motion, the depth of feature points that needs to be estimated to further obtain the spatial position information of feature points was determined. The distance of the same point was determined by observing the angle between two points. As shown in Fig. 3 (c), the images observed at two places were $\mathrm{I}_{1}$ and $\mathrm{I}_{2}$. The camera was located at $\mathrm{O}_{1}$ and $\mathrm{O}_{2}$. The feature point $\mathrm{p}_{1}$ was in $\mathrm{I}_{1}$ whereas the feature point $\mathrm{p}_{2}$ was in $\mathrm{I}_{2}$. Theoretically, the straight lines $\mathrm{O}_{1} \mathrm{p}_{1}$ and $\mathrm{O}_{2} \mathrm{p}_{2}$ intersected at a point $\mathrm{p}$, which was the position in the 3D scene determined by the two feature points. The calculation formula was as follows: $s_{2} x_{2}^{\Lambda} x_{2}=0=s_{1} x_{2}^{\Lambda} R x_{1}+x_{2}^{\Lambda} t$, where $x_{1}$ and $x_{2}$ are the normalized coordinates of the two feature points, R is the rotation matrix, t is the translation vector, and $S_{1}$ and $S_{2}$ are the depths of the two feature points to be solved. After obtaining the $S_{1}$ and $S_{2}$, the depth of the feature points under two frames can be obtained, indicating that the spatial coordinates are determined.

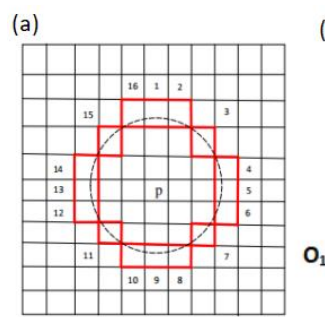

(b)

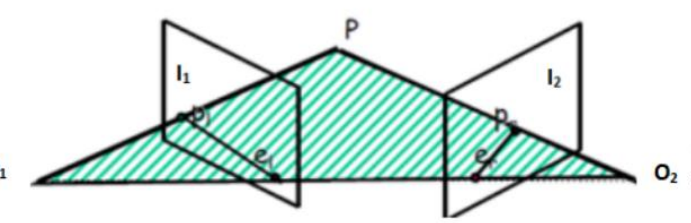

(c)

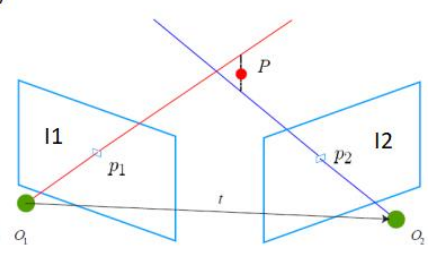

Fig. 3 Schematic diagram of augmented reality principle (a) schematic diagram of fast corner extraction principle; (b) schematic diagram of camera pose estimation principle; (c) schematic diagram of spatial position estimation of feature points;

\subsubsection{Isocenter calibration of 3D visual model}

Finding a new method to calibrate the isocenter of the 3D virtual model is necessary to ensure that the 3D virtual model is at the isocenter of the LINAC. During the design of TPS, a virtual cube model with the same size as the cube calibration module (Fig. 4(a)) was built (Fig. 4(b)). First, a square at the isocenter was created on the cross-section according to two isocentric lines. Then, the sagittal plane was adjusted to expand the upper and lower levels, ensuring that its center at the isocenter of the LINAC. The virtual cube model and model of patients were processed together and input into the 3D Slicer software. In a practical operation, interactive operation is conducted on the 3D virtual model loaded (Fig. 4 (c)) after object recognition to ensure that the virtual cube in the 3D virtual model was aligned with the cube calibration module in all directions (Fig. 4(c)). At this time, the 3D virtual model was accurately located at the isocenter .
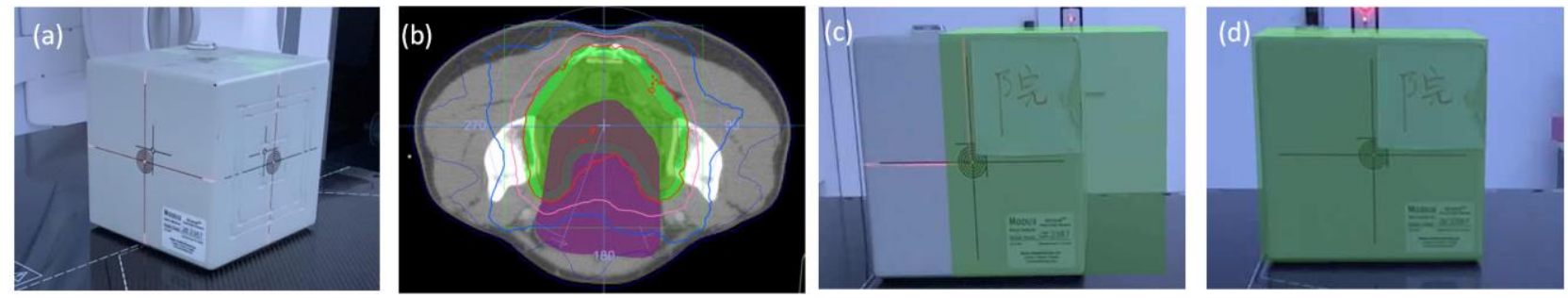

Fig. 4 isocenter calibration of 3D virtual model (a) cube calibration phantom; (b) sketch of human cross-section to build a cube; (c) identification of cube calibration phantom and loading of virtual cube; (d) aligning virtual cube with cube calibration phantom in all directions 


\subsection{Positioning of patients}

After 3D virtual model calibration, the object recognition function of the system is turned off. At this time, the 3D virtual model is fixed at the isocenter. Meanwhile, transparency adjustment is performed to display the human organ model (Fig. 5 (a)). Then, the patient is placed. First, the cube calibration module was eliminated from the treatment couch (Fig.5(b)), and the treatment couch was lowered for conventional positioning. Patients were instructed to grasp the inspiratory depth during positioning. A 3D virtual model was used as the reference object, and the treatment position of patients was adjusted (Fig.5(c)) to conform to the previous 3D virtual model on space, especially to the body profile (Fig.5(d)). The actual patient was highly consistent with the 3D virtual model, and the treatment was carried out.
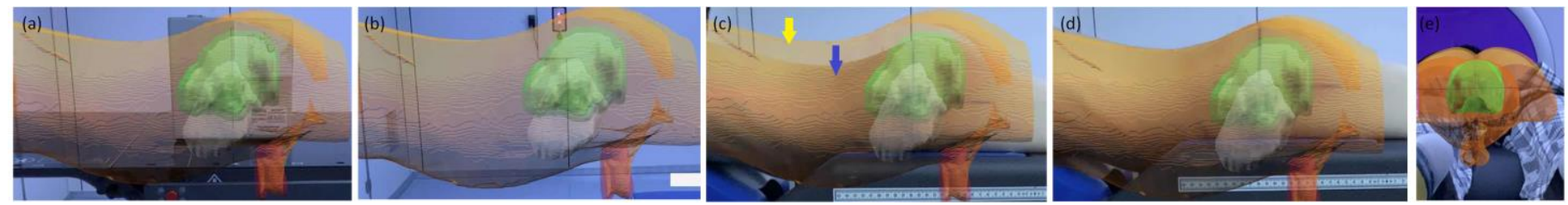

Fig. 5 (a) the internal tissues and organs are shown, PTV and CTV; (b) removal of the cube calibration phantom, adjustment of the patient's position according to the virtual model displayed by AR, and use of the 3D virtual model to align in all directions; (c) positioning of the patient according to the virtual model displayed by AR, the yellow arrow is the 3D virtual model, and the blue arrow is the actual patient; (d) In the direction of the bed side, the patient position is consistent with the 3D virtual model in all directions; (e) in the direction of the bed end, the patient position is consistent with the 3D virtual model in all directions.

\subsection{Statistical processing}

CBCT scanning results were used as the gold standard, and all relevant data were processed by SPSS 22.0 statistical processing software. Measurement data were expressed by mean \pm standard deviation ( $\bar{X} \pm$ S). Patients requiring radiotherapy on the head and neck were designated as Group A, whereas the patients requiring radiotherapy on the chest and abdomen were designated as Group B. A pairwise t-test was performed to determine the manual and AR-guided positioning errors of both groups. The test standard was $\alpha=0.05$, and statistically significant differences were observed at $\mathrm{P}<0.05$.

\section{Results}

The displacement errors of Groups A and B in the $\mathrm{x}, \mathrm{y}$, and $\mathrm{z}$ directions are listed in Table 1. The manual and AR-guided positioning errors of two groups are as follows: $-0.208-0.257$ and $-0.145-0.280 \mathrm{~cm}$ on the x-axis;-0.547-0.219 and -0.154-0.215 cm on the $y$-axis; and -0.469-0.155 and -0.214-0.09 cm on the z-axis. According to the K-S test of normality, data of Groups A and B in the $\mathrm{x}, \mathrm{y}$, and $\mathrm{z}$ directions all conformed to normal distribution.

Table 1 Positioning errors of Groups $\mathrm{A}$ and B in the $\mathrm{x}, \mathrm{y}$, and z directions $(\bar{X} \pm \mathrm{s}, \mathrm{cm})$

\begin{tabular}{lcccccc}
\hline \multirow{2}{*}{ Group } & \multicolumn{2}{c}{ Direction } & \multicolumn{2}{c}{ Y } & & Manual \\
\hline A & Manual & AR & Manual & AR & AR \\
A & $-0.010 \pm 0.295$ & $0.043 \pm 0.121$ & $-0.127 \pm 0.233$ & $0.196 \pm 0.085$ & $-0.117 \pm 0.059$ & $-0.177 \pm 0.139$ \\
A & $0.013 \pm 0.199$ & $-0.020 \pm 0.060$ & $0.160 \pm 0.072$ & $0.093 \pm 0.124$ & $-0.020 \pm 0.125$ & $-0.076 \pm 0.032$ \\
B & $0.010 \pm 0.053$ & $-0.053 \pm 0.042$ & $-0.037 \pm 0.225$ & $0.087 \pm 0.091$ & $0.083 \pm 0.172$ & $0.040 \pm 0.053$ \\
B & $0.480 \pm 0.110$ & $0.041 \pm 0.106$ & $-0.193 \pm 0.600$ & $0.173 \pm 0.045$ & $-0.613 \pm 0.180$ & $-0.097 \pm 0.155$ \\
B & $-0.170 \pm 0.242$ & $0.030 \pm 0.479$ & $-0.533 \pm 0.593$ & $-0.047 \pm 0.153$ & $-0.367 \pm 0.107$ & $-0.187 \pm 0.055$ \\
B & $0.050 \pm 0.010$ & $0.227 \pm 0.035$ & $-0.083 \pm 0.491$ & $0.220 \pm 0.020$ & $0.037 \pm 0.397$ & $-0.153 \pm 0.085$ \\
B & $-0.167 \pm 0.150$ & $-0.043 \pm 0.130$ & $0.110 \pm 0.151$ & $-0.083 \pm 0.090$ & $0.050 \pm 0.187$ & $0.150 \pm 0.246$ \\
B & $-0.040 \pm 0.113$ & $-0.070 \pm 0.095$ & $-0.340 \pm 0.345$ & $-0.277 \pm 0.095$ & $-0.413 \pm 0.386$ & $-0.043 \pm 0.133$ \\
\hline
\end{tabular}


T-test was carried out for manual and AR-guided positioning displacement errors in the $\mathrm{x}, \mathrm{y}$, and $\mathrm{z}$ directions in Groups A and B. The sample statistics of T-test are shown in Table 2, and the paired sample test of T-test is shown in Table 3.

Table 2 Sample statistics for pairwise t-test of Groups A and B

\begin{tabular}{cccccc}
\hline Group & Direction & Mode & Mean & Std.Deviation & Std.Error Mean \\
\hline \multirow{4}{*}{ A } & X & manual & 0.004 & 0.180 & 0.060 \\
& & AR & -0.010 & 0.082 & 0.027 \\
& & manual & -0.001 & 0.209 & 0.070 \\
& & AR & 0.088 & 0.140 & 0.047 \\
& & manual & -0.018 & 0.140 & 0.047 \\
& & AR & -0.071 & 0.121 & 0.040 \\
& & manual & 0.034 & 0.259 & 0.061 \\
& & AR & 0.106 & 0.248 & 0.058 \\
& & manual & -0.246 & 0.427 & 0.101 \\
& & AR & 0.002 & 0.200 & 0.047 \\
& & manual & -0.227 & 0.352 & 0.083 \\
& & AR & -0.057 & 0.169 & 0.039 \\
\hline
\end{tabular}

Table 3 Pairwise sample test for the t-test of Groups A and B

\begin{tabular}{cccccccc}
\hline Group & Direction & Mean & Std.Deviation & Std.Error Mean & $\mathrm{t}$ & $\mathrm{df}$ & $\mathrm{P}$ \\
\hline \multirow{3}{*}{$\mathrm{A}$} & $\mathrm{X}$ & 0.014 & 0.248 & 0.083 & 0.175 & 8 & 0.865 \\
& $\mathrm{Y}$ & -0.089 & 0.266 & 0.089 & -1.003 & 8 & 0.345 \\
& $\mathrm{Z}$ & 0.053 & 0.092 & 0.031 & 1.735 & 8 & 0.121 \\
$\mathrm{H}$ & $\mathrm{X}$ & -0.072 & 0.169 & 0.040 & -1.805 & 17 & 0.089 \\
& $\mathrm{Y}$ & -0.248 & 0.473 & 0.112 & -2.225 & 17 & 0.040 \\
& $\mathrm{Z}$ & -0.169 & 0.340 & 0.080 & -2.113 & 17 & 0.049 \\
\hline
\end{tabular}

(The P value was derived from the bilateral test.)

According to Tables 2 and 3, for group A data analysis, the errors of manual positioning and AR-guided x-axis positioning were $-0.176-0.184 \mathrm{~cm}$ and $-0.092-0.072 \mathrm{~cm}$, respectively, but the difference was not statistically significant $(\mathrm{t}=0.175, \mathrm{P}=0.865>0.05)$. The $\mathrm{y}$-axis errors of manual and AR positioning were $-0.219-0.199 \mathrm{~cm}$ and $-0.052-0.228 \mathrm{~cm}$, respectively, with no significant difference $(\mathrm{t}=$ -1.003, $\mathrm{P}=0.345>0.05$ ). The z-axis error range of manual positioning and AR positioning were $-0.158-0.122 \mathrm{~cm}$ and-0.192-0.05 $\mathrm{cm}$, respectively, with no significant difference $(t=1.735, P=0.121>0.05)$. The positioning errors of patients who needed AR-guided head and neck radiotherapy were not significantly lower than those of patients who needed manual positioning, but the positioning errors of AR-guided and manual positioning remained within a small range.

For the group B data, the positioning errors of manual and AR-guided positioning on the $\mathrm{x}$-axis were -0.225-0.293 cm and-0.142-0.354 $\mathrm{cm}$, respectively, but the difference was not statistically significant $(\mathrm{t}=-1.805, \mathrm{P}=0.089>0.05)$. The $\mathrm{y}$-axis errors of manual positioning and AR positioning were $-0.673-0.181 \mathrm{~cm}$ and $-0.198-0.202 \mathrm{~cm}$, respectively, with a significant difference $(\mathrm{t}=-2.225, \mathrm{P}=0.04<0.05)$. The positioning errors of z-axis under manual and AR guidance were in the range of -0.579-0.125 and-0.226-0.112 cm, with significant difference $(t=-2.113, P=0.049<0.05)$. For patients who needed chest and abdominal radiotherapy, the positioning errors of $y$-axis and z-axis under AR guidance were significantly lower than those of manual positioning, but on the x-axis, AR-guided positioning errors were not significantly lower than manual positioning errors.

For patients undergoing chest and abdomen radiotherapy, the effect of AR-guided positioning error reduction was significantly greater than that for patients undergoing head and neck radiotherapy, and the effect of AR-guided positioning error reduction was more obvious 
for patients undergoing chest and abdomen radiotherapy.

\section{Discussion}

An AR visual image guided radiotherapy set-up system was proposed in this study. It helped realize noninvasive and nonradiative 3D virtual modeling for the radiotherapy plan. The 3D virtual model and real space were fused by AR technology, which provided certain guidance for positioning during radiotherapy. Through an experiment, the present study showed that the proposed AR system was feasible in radiotherapy positioning. It decreased positioning error effectively and completely eradicated the occurrence of treatment errors. The AR visual image-guided radiotherapy set-up system is a type of surface guided radiotherapy. The experimental results obtained in the present study were similar to those obtained with SGRT. The positioning error of the proposed system was approximately 3 mm[11].

For patients requiring radiotherapy on the head and neck, AR-guided positioning error was not decreased significantly compared with manual positioning error. This finding might be related to the presence of few soft tissues in the head and neck, which are not influenced by breathing movements and are only slightly influenced by small changes in the body ${ }^{[12]}$. However, the AR system can keep the positioning error within a small range.

For patients requiring radiotherapy on the chest and abdomen, AR-guided positioning errors on the y-axis and z-axis were significantly lower than manual positioning errors. Patients needing radiotherapy on the chest and abdomen often need their position fixed by using thermoplastic film and vacuum pad. The thermoplastic film fixation often makes radiotherapy markers on the thermoplastic film ${ }^{[13]}$, whereas the vacuum pad fixation needs radiotherapy markers on the body of patients and the vacuum pad. Marking lines serve as the main references for manual positioning. However, manual positioning based on these marking lines is inaccurate[14]. First, the positioning marking errors on the body of patients are high due to the relatively loose skin of some patients or to their inaccurate lying position during treatment ${ }^{[15][16]}$. Second, the width of marking lines reaches $0.3-0.5 \mathrm{~mm}$ after repeated tracing, and a certain error is present in the manual alignment with marking lines. Moreover, marking lines on patients requiring radiotherapy on the chest and abdomen can have certain positioning errors, because of breathing activity and body changes that occur during radiotherapy, especially for overweight patients. AR-guided radiotherapy positioning does not require a marking line on patients.

However, the proposed AR-guided radiotherapy positioning system still needs improvement. First, To ensure the isocentric placement of the 3D virtual model, the cube calibration module is used for isocentric calibration with the virtual cube. However, in the calibration process, the virtual cube loaded by object recognition technology cannot be automatically matched to the cube calibration module. It relies on the AR interactive system for operation, but this system has low efficiency and needs improvement in terms of accuracy. To solve this problem, we will introduce ICP algorithm for correction[17] and complete automatic matching of 3D virtual model to isocenter ${ }^{[18][19]}$. Second, the system we proposed is used in radiotherapy patient positioning, and other functions, such as real-time monitoring during radiotherapy, are not considered. In the future, we will introduce real-time monitoring function into the system.

Future studies will address the abovementioned problems to realize the intelligence and multiple functions of the AR-guided radiotherapy positioning system. We will integrate deep learning and neural network into the AR-guided radiotherapy positioning system to conduct automatic matching between the 3D virtual model and the real model to increase positioning efficiency. In addition, a matching algorithm will be integrated to remind clinicians and researchers of the distance error between the practical body position of patients and 3D virtual model by real data.

\section{Conclusions}

A statistically significant difference exists between AR visual image-guided radiotherapy set-up system and manual positioning errors. AR visual image-guided radiotherapy setup error is smaller than the manual positioning error, especially for patients requiring chest and abdomen radiotherapy. The AR-guided radiotherapy positioning error can be kept within a small range for patients requiring head and neck radiotherapy. 


\section{Ethics approval and consent to participate}

The present study was approved by the Clinical Ethics Committee of Second People' s Hospital of Changzhou of Nanjing Medical University (Changzhou, China).

\section{Consent for publication}

Not applicable.

\section{Availability of data and materials}

The datasets used and/or analyzed during the current study are available from the corresponding author on reasonable request.

\section{Competing interests}

The authors declare that they have no competing interests.

\section{Funding}

This work was supported by High-Level Medical Talents Training Project of Changzhou (grant no: 2016CZLJ004), Changzhou Key Laboratory of medical physics, Jiangsu Province, China (grant no. CM20193005), Youth Project of Changzhou Municipal Commission of Health and Family Planning (grant no:QN201718) and Jiangsu Province postgraduate scientific research innovation plan in 2020(grant no.KYCX20_1421).

\section{Authors' contributions}

The manuscript was written through the contributions of all authors. LCY and NXY participated in the design of the study, carried out the study, performed the statistical analysis, and drafted the manuscript. LZD, HM, SJF and LT helped to carried out the study. XK and SJW reviewed and edited the manuscript. All authors read and approved the fifinal manuscript.

\section{Acknowledgments}

The author would like to thank Mr. Dai Jianrong from the Radiotherapy Department of Cancer Hospital Chinese Academy of Medical Sciences for his help and the ChangZhou LeiMu company for its technical guidance.

\section{References}

[1] Hussain Raabid,Lalande Alain,Guigou Caroline,Bozorg-Grayeli Alexis. Contribution of Augmented Reality to Minimally Invasive Computer-Assisted Cranial Base Surgery.[J]. IEEE journal of biomedical and health informatics,2020,24(7).

[2] Jud Lukas,Fotouhi Javad,Andronic Octavian,Aichmair Alexander,Osgood Greg,Navab Nassir,Farshad Mazda. Applicability of augmented reality in orthopedic surgery - A systematic review.[J]. BMC musculoskeletal disorders,2020,21(1).

[3] Z. Zhang, D. Weng, H. Jiang, Y. Liu and Y. Wang, "Inverse Augmented Reality: A Virtual Agent's Perspective," 2018 IEEE International Symposium on Mixed and Augmented Reality Adjunct (ISMAR-Adjunct), Munich, Germany, 2018, pp. 154-157.

[4] Talbot J,Meyer J,Watts R,Grasset R. A method for patient set-up guidance in radiotherapy using augmented reality.[J]. Australasian physical \& engineering sciences in medicine,2009,32(4).

[5] Varian Medical Systems.Calypso system operator's manual[R].PaloAlto,CA:Varian Medical Systems,2013.

[6] A M Franz,D Schmitt,A Seitel,M Chatrasingh,G Echner,U Oelfke,S Nill,W Birkfellner,L Maier-Hein. Standardized accuracy assessment of the calypso wireless transponder tracking system[J]. Physics in Medicine and Biology,2014,59(22).

[7] Twyla R. Willoughby,Patrick A. Kupelian,Jean Pouliot,Katsuto Shinohara,Michelle Aubin,Mack Roach,Lisa L. Skrumeda,James M. 
Balter,Dale W. Litzenberg,Scott W. Hadley,John T. Wei,Howard M. Sandler. Target localization and real-time tracking using the Calypso 4D localization system in patients with localized prostate cancer[J]. International Journal of Radiation Oncology, Biology, Physics,2006,65(2).

[8] Stanley DN, McConnell KA, Kirby N, Gutiérrez AN, Papanikolaou N, Rasmussen K. Comparison of initial patient setup accuracy between surface imaging and three point localization: a retrospective analysis. J Appl Clin Med Phys. 2017;18:58-61.

[9] Cravo Sá A, Fermento A, Neves D, et al. Radiotherapy setup displacements in breast cancer patients: 3D surface imaging experience.Rep Pract Oncol Radiother. 2018;23:61-67.

[10] Zhao Hui,Williams Ned,Poppe Matthew,Sarkar Vikren,Wang Brian,Rassiah-Szegedi Prema,Huang Y Jessica,Kokeny Kristine,Gaffney David,Salter Bill. Comparison of surface guidance and target matching for image-guided accelerated partial breast irradiation (APBI).[J]. Medical physics,2019,46(11).

[11] Laaksomaa M , Sarudis S , Rossi M, et al. AlignRT and Catalyst in whole-breast radiotherapy with DIBH: Is IGRT still needed?[J]. Journal of Applied Clinical Medical Physics, 2019, 20(3):97-104.

[12] Mori S, Hara R, Yanagi T, et a1. Four dimensional measurement of intrafractional respiratory motion of pancreatic tumours using a 256 multi-sljce CT scanner[J]. Radiother Oncol, 2009, 92(2) : 231.237.

[13] Ohira S, Ueda Y, Nishiyama K, et a1. Couch height-based patient setup for abdominal radiation therapy(J]. Med Dosire, 2016, 41(1) : 59-63.

[14] Wang H, Wang C, Tung S , et al. Improved setup and positioning accuracy using a three-point customized cushion/mask/bite-block immobilization system for stereotactic reirradiation of head and neck cancer[J]. Journal of Applied Clinical Medical Physics, 2016, 17(3):180-189.

[15] Petillion S, Verhoeven K, Weltens C, et al. Efficacy and workload analysis of a fixed vertical couch position technique and a fixed-action-level protocol in whole-breast radiotherapy.[J]. Journal of Applied Clinical Medical Physics, 2015, 16(2):279-290.

[16] Riboldi M , Orecchia R , Baroni G . Real-time tumour tracking in particle therapy: technological developments and future perspectives.[J]. Lancet Oncology, 2012, 13(9):e383-e391.

[17] Q. Gao, T.R. Wan, W. Tang, L. Chen, "A Stable and Accurate Marker-less Augmented Reality Registration Method", In: 2017 International Conference on CYBERWORLDS, 20-22, 2017.

[18] Shi,Liu,Han. Improved Iterative Closest Point(ICP) 3D point cloud registration algorithm based on point cloud filtering and adaptive fireworks for coarse registration[J]. International Journal of Remote Sensing,2020,41(8).

[19] Kyeong-Beom Park,Sung Ho Choi,Minseok Kim,Jae Yeol Lee. Deep learning-based mobile augmented reality for task assistance using 3D spatial mapping and snapshot-based RGB-D data[J]. Computers \& Industrial Engineering,2020,146. 

Figures

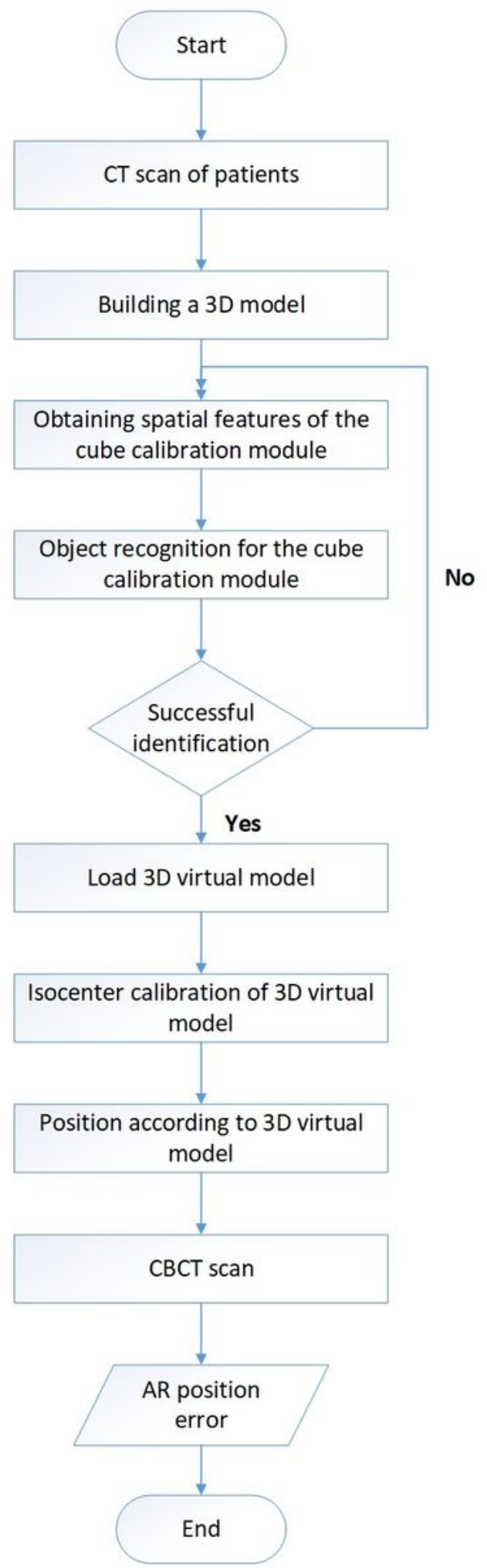

Figure 1

Manufacturing process of the system 


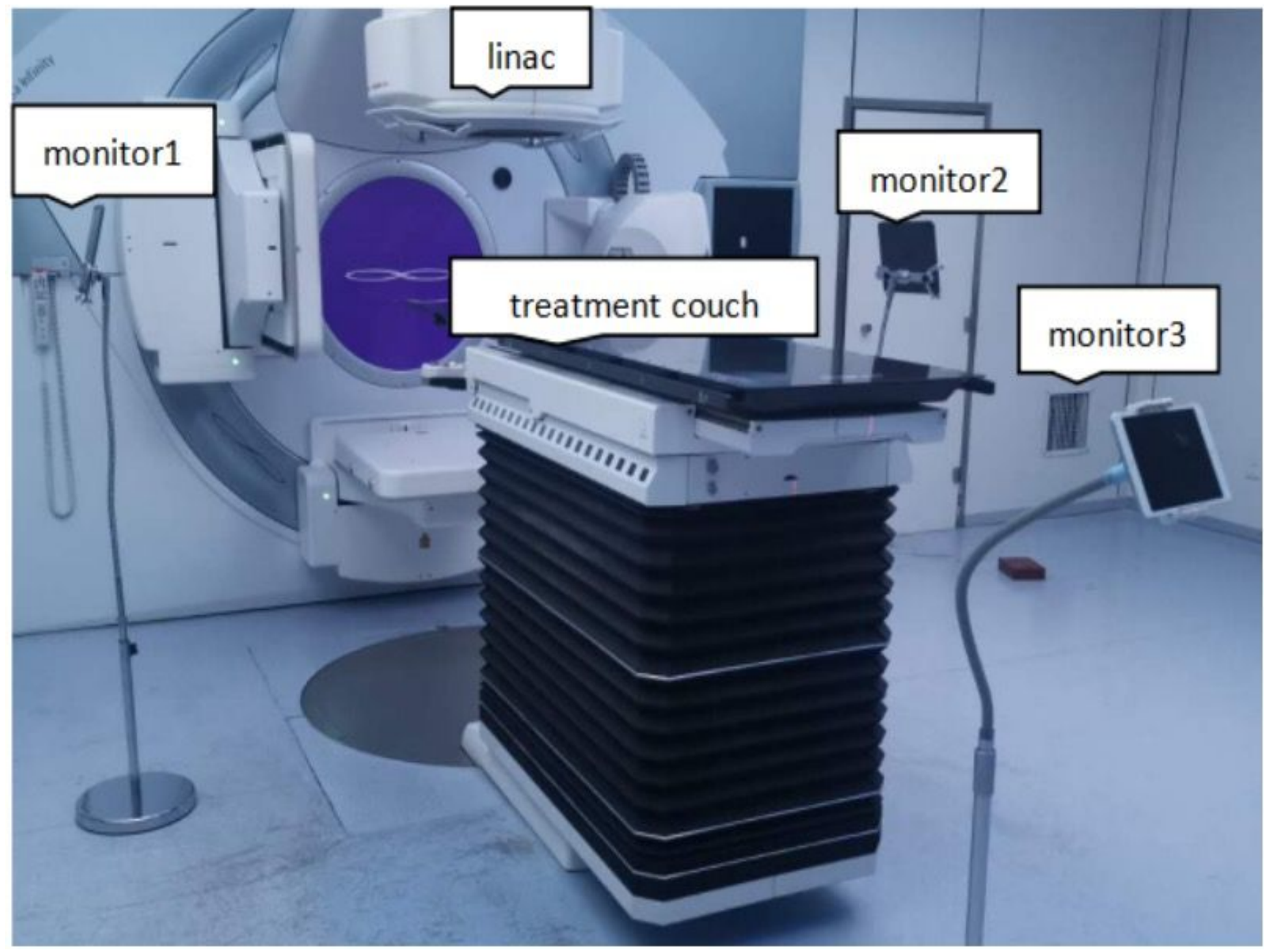

Figure 2

Overall system setting

(a)

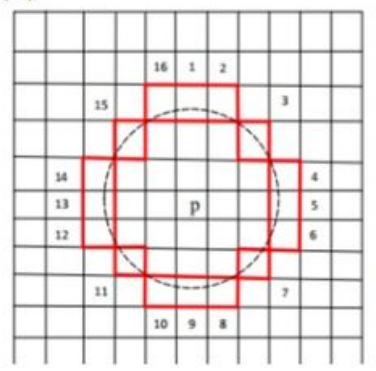

(b)

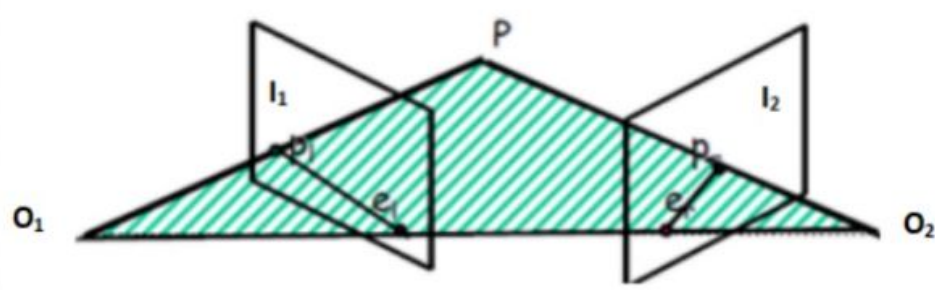

(c)

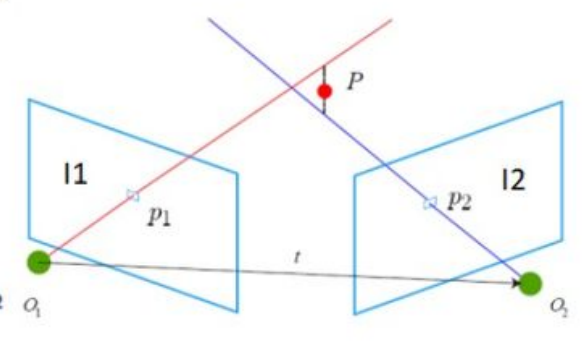

\section{Figure 3}

Schematic diagram of augmented reality principle (a) schematic diagram of fast corner extraction principle; (b) schematic diagram of camera pose estimation principle; (c) schematic diagram of spatial position estimation of feature points; 

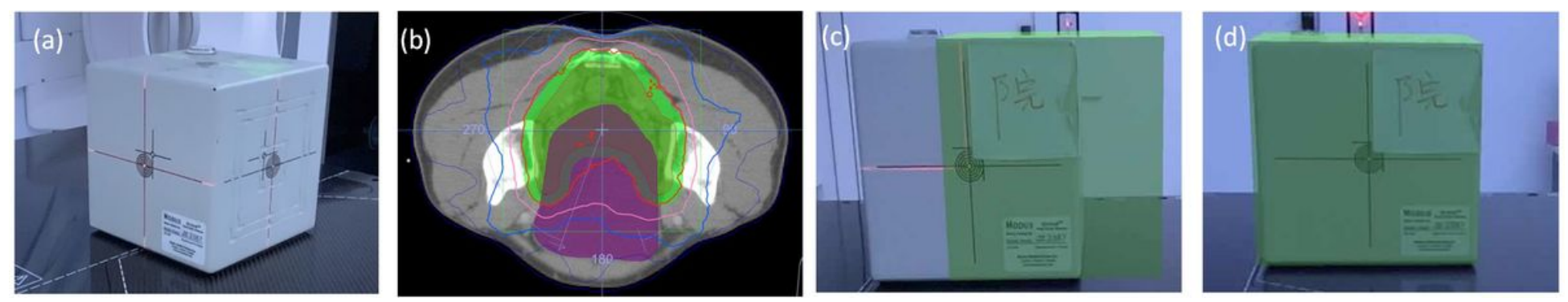

\section{Figure 4}

isocenter calibration of 3D virtual model (a) cube calibration phantom; (b) sketch of human cross-section to build a cube; (c) identification of cube calibration phantom and loading of virtual cube; (d) aligning virtual cube with cube calibration phantom in all directions
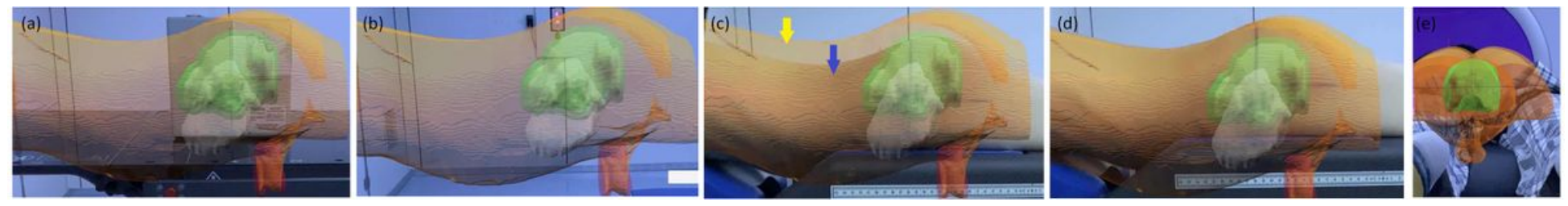

\section{Figure 5}

(a) the internal tissues and organs are shown, PTV and CTV; (b) removal of the cube calibration phantom, adjustment of the patient's position according to the virtual model displayed by AR, and use of the 3D virtual model to align in all directions; (c) positioning of the patient according to the virtual model displayed by AR, the yellow arrow is the 3D virtual model, and the blue arrow is the actual patient; (d) In the direction of the bed side, the patient position is consistent with the 3D virtual model in all directions;

(e) in the direction of the bed end, the patient position is consistent with the 3D virtual model in all directions. 